\title{
Essence, Ethos, and Early English Texts: The Old New Spelling Wars
}

\author{
Donald Beecher \\ Carleton University
}

The matter of modernizing and repunctuating old texts is, in a sense, merely an option among options - ultimately a question of taste and aesthetic preferences. Arguably, it is a means for rendering the remote and formidable into a more accessible form, but for some, the costs of such procedures are too high. Advocacy for old spelling and non-editorial intervention in such texts is predicated upon arguments of historicity, authorial intentionality, and a unique quality of reading experience, all of which are forfeited or compromised by modernization, not to mention the pedagogical value of such editions in training students to read from the originals. The modernizing approach 
is guided by the principle of mediating the past to a modern readership. The other is informed by the ideal of exposure to earlier cultures by mastering the skills requisite to read, presumably, as "they" read, and, more conjecturally, to experience texts as "they" experienced them. These are wellknown positions, both putatively laudable. But the question remains for the prospective editor whether one approach or the other should be adopted, given that, ipso facto, a text cannot be presented both ways at once. It is, in fact, useless to even imagine compromises between the two, some of this and less of that, for such editions will inevitably fall foul of both camps rather than placate or satisfy. Moreover, to increase the agony, feelings about the matter can run very high: in the old spelling camp, about the limited usefulness or validity of modernized texts; among modernizers, that another generation of students will be punished by being inflicted with arbitrary Elizabethan orthography and meaningless printing conventions that only stand in the way of the message. There the debate might end as a matter of pragmatic choice. Yet the impasse implies, nevertheless, questions about the nature of texts and why we read them. Old spelling prioritizes reading as an antiquarian enterprise, whereas modern spelling prioritizes the story as an independent Gestalt realized in the mind's eye. Is it meaningful to ask, then, how the exposure to the antiquarian actually participates in the realization of that Gestalt, or if the formatting and printing conventions are an essential part of the reading experience? These are more difficult questions, for early texts were not antiquarian to their original readers. In brief, how important is old format to reading old texts when format was neutral for them but is antiquarian and distancing for us? Inversely, what is lost of the "intended" message when it is 
transmitted through modern spelling, formatting and punctuating conventions?

In the editorial presentation of such texts, there are but three distinct possibilities, among which both editors and publishers must choose. Either the text is presented in photographic facsimile, such as Elizabethan black-letter (typical of all the prose fiction productions of that era) with all its typographical idiosyncrasies intact (see McKerrow, 1967, p. 297), or it is presented in modern typefaces in a diplomatic transcription both for spelling and punctuation, but perhaps with abbreviations expanded, or it is modernized in some optimal way that regularizes all the non-substantive anomalies of Elizabethan style while recording every substantive aspect of the writing. The philosophical implications of only these choices can exfoliate and luxuriate into speculations about the nature of texts, readers, and the sign systems that convey literary meanings.

\section{Printing Conventions and Narrative Meaning}

Regarding the facsimile, Elizabethan black-letter or gothic type was in common use among the many printers of popular literature until well into the seventeenth century. There was nothing out of the ordinary in its design, and when it was properly inked, gracefully and conventionally spaced, and not obscured by shadowing from ink on the opposite sides of the pages (rarely uniformly so in most early productions), it could be quite elegant. Arguably, for some, such type is as much a part of the Elizabethan aesthetic as the stories themselves and comparable to the visual flow of the musical notation on the 
pages of their part books. Thus, to enjoy the fullest dimensions of the Elizabethan reading experience, the reader must simply master the skills involved in decoding the characters and conventions of the age. If black-letter was their reality, it should be ours as well.

To presume, however, that there is something intrinsic to the message embedded in this medium is an assertion of a different order. Incontestably, the ability to read early typefaces as fluently as the Elizabethans must have done is a useful skill, and perhaps one worth attaining in its own right. But while typefaces may have their aesthetic appeal, there is a harder argument to be made that the meaning of the récit itself is nuanced by its typographical presentation, whether in blackletter, or Garamond, Caslon, Palatino, or Times Roman. Inversely, it might be argued that as the most common and current typeface of the Elizabethan period, black-letter should be replaced by the most common and current contemporary type because that which least interferes aesthetically or eccentrically with the rendition of words into mind concepts and images is best. After all, there is no evidence that the Elizabethans, in using their typefaces, intended to be antiquarian or aesthetic beyond meeting the conventions of the typeset page with its justified margins. Moreover, there is no evidence that page design was chosen in accordance with the ethos of the fiction itself, as witnessed by the choice of a common title page design for books ranging from novelle, to religious treatises, to medicine, as in the case of the David and Moses title page used by Riche's printer for the Farewell to Military Profession (1581). 
But there is an altogether more compelling kind of reasoning to explore in separating the message from the printed medium: reasoning derived from "folk psychology," according to which cognitive philosophers have been persuaded (if not compelled) to accept that other minds function like our own with regard to the reading of psychological environments, such as the intentional states in other persons, and in orienting themselves in their material environments - which is much of what we do in the reading of fiction. We are oriented toward searching for clues concerning the beliefs and motivations of persons described and how those mind states impinge upon the destinies of others in accordance with our own desires for those characters. On this score, we must assert that Elizabethans were story-tellers in the same generic sense that we are story-tellers, and that their words are directed to our cognitive and emotional capacities to convert signs to mental events that represent meaningful and suspenseful sequences to us. Such sequences we assemble into felt qualities of imaginative experience, much as we do with contemporary stories. From that perspective, the question remains whether anything essential to that narrative experience can be determined by the conventions of the signing system through which it is conveyed to the imagination. It is not an easy question to answer. Another way of asking that question is whether the formatting of the printed page has intentional or passive designs upon our formulation of the story events. This is to rephrase the opening question in teleological terms by emphasizing the function of the printed page as a vehicle to narrative - as the most efficient means known, whatever the historical period, for relaying the representation of equivalent-to-reality experiences through typographical and 
orthographical signs. The page as it appears then becomes merely an acknowledgement of a message system in developmental flux searching for the optimum conventions for the transmission of stories in the absence of their narrators. That Elizabethan story-tellers so often entered into their stories as the voices behind their narratives, talking to and coaching readers, is itself an interpretation of the intended uses of the printed page. By this reasoning, the narrative experience beginning in consciousness and ending in consciousness as a representational series of events is the prioritized cultural phenomenon; the rest is entropy, a mere drag on the line of communication.

In contradistinction, there is a case to be made for the printed page as a cultural artefact in its own right, as a particularized form of information transmission, the components of which are an essential part of the message. If the Elizabethan printers made orthographical mistakes, they are interesting in themselves, perhaps telling ambiguities, perhaps potential puns, perhaps revelatory of the Freudian life of the compositor. Spacings that lead the eye from symbol to symbol in elliptical ways possess a kind of latent intentionality. At the least, all such features provide unique acts of recognition to be acknowledged in counterpoint to the mental actualization of fictional events. The literary page is potentially performative in much the same way that musical pages are putatively performative, on the assumption that the sign systems of music, through their very disposition on the page, contribute to a sense of the ethos of the work. In that regard, the same story or composition is a different work in accordance with the variations of the layout of the music or text. Or is it? The issue is particularly acute for those practitioners of early music who 
consent to perform only from facsimiles on the assumption that the colouring of the notes, the pre-modern time signatures, and the spacing on the pages speak volumes about how the music feels, and hence how it is to be performed. That frame of mind went so far as to assume some important intentionality in a set of part books in which three lines had repeat marks at the ends of the phrases and two did not. Ferrabosco, then, by a certain perverse logic, genuinely intended for consorts to collapse in confusion at the ends of those phrases on the assumption that a faulty medium was part of the message. But if common sense suggests that a uniform musical message be found in spite of the faulty copywork, can the case be made for a uniform musical message as early compositions are transcribed into the exact equivalent musical conventions of any successive age? That debate is far from over. Likewise, can it then be said that a narrator of fiction seeks a common message and experience across and through the accidents and idiosyncrasies of the printing process? That white notes in certain time signatures in the Renaissance mean exactly what we mean today by black notes in a reduced time signature finds no persuasion with the purists, even though the mathematical equivalents are identical ; white notes for them simply feel different than black notes, just as the long s may feel different from the short one, even though it has been eliminated from modern typefaces. Moreover, unedited early texts bear a resemblance to the challenges in performing from facsimile, whereby all the scribal errors must be calibrated in the memory of the performers and spontaneously corrected in order to realize a satisfactory performance. Are copy errors performed for the sake of musical authenticity? If not, then someone must be an editor, whether it is the scholar or the performer. Likewise, if, in reading fiction, 
"someone" must correct and interpret the signs, by what criteria are those "corrections" made and is there an ideal text that all readers must find in order to arrive at an optimum actualized experience? Such words as "optimum" will make the well-trained relativist bristle, but the argument remains worth an additional philosophical fillip. It inheres in the notion of a common message that guides the editorial interventions of the textual mediator, that there is an "essential" dimension in the narrative inherent to the story logic that resists all forms of external colouration, an essence that guides the decisions of the editor. Or again, it is the conviction that there is an inevitable cognitive reconstruction of event, voice, and experience to be found that transcends the typographical conventions of any age through which it might pass.

\section{Rationales for the Modernized Text}

The first order of concession to the modern in the mediation of a common message is the diplomatic transcription which employs a modern typeface, but which retains nearly all the original conventions of orthography and punctuation. It thereby sheds the alienating look of the Elizabethan page and all else that might constitute a part of the authentic Elizabethan reading experience, including a straight back leather chair, a book still bound in its original binding, and the letters swimming in the flicker of candlelight. (Quite apart from its absence in the modern reading brain, authenticity is lost in other terms very early on.) The diplomatic text is deemed a necessary concession simply because modern publishers are not equipped with antiquarian typefaces. What really matters, after all, are the "proto-substantives," matters of spelling and 
punctuation. Yet the concessions have begun. By the logic of the true purist, the book without its original typeface is already a whore, thereby disqualifying all forms of editorial intervention or modernizing strategies; all editors may break camp.

This is an ungrateful chip-away approach to the antiquarian ideal, and the slippery slope has its weaknesses as a debate strategy. Nevertheless, if the purpose of the text is to know the work in the form in which it was written by the author as a means of attaining a mystical contact with the man and his mind, defeat sets in early, for the Elizabethan compositors had no respect for the orthography of authors on the whole and tended to spell according to their own conventions. Or, as it has been argued, "responsibility for punctuation (as for spelling) was forced upon the printinghouse compositors by the erratic punctuation employed by most writers" (Dawson and Kennedy-Skipton, 1966, p. 18). This is to presume that compositors were editors, themselves looking for the standardized message among the random signs. Either way, the printing house variants become particularly clear in works having more than one compositor, resulting in contrasting sections in the book. Moreover, compositors resorted to variants upon their own conventions in accordance with the disposable space within lines. They used macrons and ampersands, abbreviations of the "ye old curiosity shop" variety, and the doubling of letters or even the addition or deletion of words to justify margins or to make texts join at the ends and beginnings of gatherings. The exigencies of book layout dictated many such orthographical and spacing variants in an effort to make a pretty book, not to mediate the story in an Elizabethan way. This is standard news with all bibliographers and need not be belaboured here, except to underscore that in 
the fine tunings of word presentation, authors - who rarely spelled consistently themselves, and often wrote their own names several different ways - are largely obscured. There is, moreover, an argument to be made on further historical grounds that both orthography and punctuation were entirely personal and eccentric during this period, but that standardization was gradually making progress, particularly in the latter part of the seventeenth century. Hence, there is less reason to maintain eccentricities that were, in any case, slowly giving way to the standardizations that prefigured our modern conventions.

In a more immediate sense, there is the text itself and the argument, on cognitive grounds, for the construction of the common message. Here, from John Dickenson's Greene in Conceit, a charming domestic tragedy published in 1598, is a sentence more or less selected at random, illustrating the kinds of differences separating then from now:

But when Iockie (which would not cowardly give ouer hauing thus entred) continued his discoueries, still furnisht with more friendly opportunities, \& did oft constantly offer upon the hazard of ye whip, to make his master eye=witness of that, whereof his eares deignd no acceptace; Giraldo at last deeply reuoluing in his pensiue thoughts the boies large proffer, and much desirous to know at full the states of his own forehead, wherein he seemd to feele already some alteration, agreed to his request, waiting a couenient time; \& for ever sorrowd to remember (p. 102).

In these matters, the reading eye can become quite adept, converting i's to j's and u's to v's, filling out the ampersands and macrons, turning ye to the, dropping the e in "eares" and adding one to "deignd," and gliding over the pointless semicolons while adding the necessary comma between "cowardly give over" and 
"hauing thus enterd." This is easy enough and hardly merits changing; any undergraduate can master the skills. But if the editor is going to transcribe the text, thereby altering it from black-letter to modern type, what, then, is violated in spelling "boies" as boys and "reuoluing" as revolving? It is an academic question, because what is ancient to the modern eye was not intended to look antique to the Elizabethan eye. Words in that spirit as signifiers might as well be rendered into their standard format in order to call the least attention to themselves as words, because what Elizabethans meant by "boies" as a signifier differs not an iota from what we mean by "boys." If there are differences, it is not in the spelling, which was itself largely arbitrary, for "boys" was also a common Elizabethan spelling of the word.

Such a procedure, of course, strands all words having no modern equivalents or which still exist but whose meanings have changed, as in the case of "to revile their silly father." The facsimile edition can offer no help except through a glossary, whereas the diplomatic edition may conceivably have bottomof-page glosses, as in a fully-modernized critical edition. But glosses in some form are essential, for otherwise the reader is led astray by his or her own semantic shortfall - the first editorial concession that opens the entire editorial war chest. Every Elizabethan recognized the meaning of "silly" as a combination of pitiful, helpless, simple, poor or frail - all of which are legitimate pre-1600 meanings — but most modern readers are inclined to impose the anachronistic meaning of "foolish," the first recorded usage of which dates only to 1856. The gloss signals two sides to the entire debate: that editorial intrusion is essential to directing readers toward original meanings, and that, nevertheless, the misleading original words 
are inviolable. The distinction inheres in the semantic distinctness and authority of the word that does not inhere in the vagaries of orthography or punctuation. The word is substantive; orthography is not. But those remain fighting words. I am opining that meaning alone matters, and that that which alters meaning is all that needs to be taken into consideration. The onus hence lies upon the antiquarians to demonstrate that in the construction of meaning, orthography, book design, and printing conventions have an empirically demonstrable part to play. On the assumption that it was the Elizabethan writer's intention that as little as possible stand between the necessary signs and their most facile conversion into a reading experience, the editor can interfere with impunity in rendering the text into those forms that make the relation of meanings most readily available to the reader according to the most familiar conventions. Broken letters, press variants, orthographical conventions, errors, and printing house practices may be suppressed; they are materials of value only to those who wish to study the ways of compositors and printers for historical reasons. To those ends, all surviving early English books are available not only in their remaining copies or in facsimile, but on microfilm and on-line.

The matter of original pointing is subject to the same kinds of arguments, but corrective repointing may involve a greater interpretative intrusion upon the original document and not merely a simple equivalence. The question turns around the uses and meanings of punctuation, for clearly these signs have intentions in subdividing long and sometimes breathlessly compound units of syntax into smaller groupings according to a hierarchy of interruptions from the light comma to the full stop, while incorporating a frequent use of 
parentheses, semicolons, and a baffling use of full colons, according to conventions taken over largely from Latinate pointing. A primary consideration is that, "English schoolboys of the sixteenth century studied Latin, not English. And because Latin had been studied continuously since ancient times and was the language of learning, its syntax, grammar, and spelling were established and relatively immutable" (Dawson and Kennedy-Skipton, 1966, p. 16). It was only natural that Latin conventions would be employed analogously in an effort to stabilize English usage. Examples abound, and again, Greene in Conceit is a source as apt as any. There, one finds examples of lengthy sentences, as much as a page long, subdivided by a half dozen or more colons and semicolons, as well as numerous commas and parentheses, in ways that defy a clear sense of weighting and priority. There are instances in which the colons are the equivalent of periods, in others of commas, and still others of nothing at all. For a short example, the following may serve: "Breach of wedlocke had beene in her a crime inexpiable: but where the state and person of the offendour is changed, there likewise the qualitie of the offence is altered: nor can the crime bee so directly pertinent to you, (which being a child must obey) as to Theodoro, which (being your father) might and did commaund" (p. 89). The first question is whether the conventions of modern punctuation are impoverished by comparison with the Elizabethan, or whether the use of double points is simply obsolete, arbitrary, and completely absorbed by more rationalized practices. The second question is whether they are cues for oral reading, representing different qualities of breathing and phrasing, the elimination of which would hide important evidence concerning the oral presentation of these works in a culture far closer to its oral traditions. If the colon is 
nearly a full stop but not altogether so, then the sense of the syntax maintains clear divisions, yet flows on more seamlessly from phrase to phrase in the soft punctuated manner in which we actually speak. The prospects are tantalizing, but the evidence remains inconclusive. Arguably, in a utilitarian sense, the sentence above from Dickenson is more readily accessible to the modern reader when it is repunctuated with commas and periods. Arguably, too, as a read performance, no distinctions between the two pointings are perceptible. Even historically, the notion of the phrased text is difficult to defend. "Attempts have been made to analyze the dramatic punctuation found in the First Folio edition of Shakespeare, but these were largely futile because they were based on the assumption that the punctuation was Shakespeare's own rather than, as it is now held to be, the printers" (Dawson and Kennedy-Skipton, 1966, p. 17-18). Yet the argument lingers that to remove the original pointing and to impose modern conventions of punctuation and capitalization is to remove a precious record of the text as phrased in the author's mind, or as a text shaped for auditory reception. The statement has an instant appeal to our sense of duty to the past, even though performance differences are imperceptible, orthography in the period was an ad lib science, compositor's pointing replaces the author's, and Latin practices intruded upon English usage. To the degree that these reservations and qualifications undermine the substantive value of typographical and orthographical conventions, they justify the kind of conservative modernization that enhances the reception of narrative meanings. 


\section{Mediating Universal Experience}

This is to assume that there is only one common narrative experience to be had for readers of any age, and that it must be found through and in spite of the conventions of presentation. That nostrum will inevitably prove controversial, and any argument in defence of modernization heads toward this putative Charybdis. Yet a direct argument inheres, that a common and necessary sense must be extracted from signs by all readers of any age in order to make a successful conversion of the text into experience. In effect, the Elizabethans had to find the same narrative logic that we seek in rendering phrases into lived mental events. This is because, in phylogenetic terms, all cultural and historical differences aside, their minds were identical to our own and constructed meanings in precisely the same way. Thus, what matters is no longer a question of what Elizabethans may have experienced because of familiarity with their printing house conventions, but what they had to perform mentally in the recovery of narrative logic in relation to the world. They were also interpreters of signs, in seeking the representational qualities of imagined events, characters, and their motivations. They were hermeneutes in their search for deduced meanings through their processes of inference. The current discussion is not addressed to the construction of critical understanding, but merely to the transition from sign and phrase to mental representation (whatever the vagaries and personalization of that experience) in the construction of immediate cognitive meanings. The question is whether, in that process, they shed the signs, or incorporate the aesthetic distinctiveness of sign systems as part of the meaning making process. My argument is that, on the whole, the mind separates cleanly between the accidents of book presentation and the 
reconstruction of narrative elements. In this regard, among all the editorial contributions to the modernized text, only punctuation is in question. Here are to be found the greatest hesitations, whether it is a feature of meaning in itself, or subservient to the intrinsic logic of the narrative récit in more or less efficient ways. One working experiment in establishing that point is to take a representative passage from sixteenthcentury prose and to have, let us say, three modern readers interpret it through the punctuation in order to highlight its intrinsic logic. Would it stand to reason that if all three readers repunctuated it in the same way, or nearly so, that a common order of meaning has been perceived, and that this order must also have been located by the successful Elizabethan reader?

As a partial test of my hypothesis that the same sentence logic will be recovered by different readers regardless of the punctuation of the original, consider a passage from "Of Apollonius and Silla," the second story in Barnabe Riche's Farewell to Military Profession, chosen because it is the source for Shakespeare's Twelfth Night and thus one of the most frequently-edited and modernized pieces of Elizabethan prose fiction. The sample is some 18 lines long, beginning, "In this manner she travelled to Constantinople..." (1992, p. 187). I compared versions from the 1960s by Alice Griffin and by T.J.B. Spencer after preparing my own modernized edition of the Farewell. Tellingly, all three editors subdivided Riche's one long sentence into five, at precisely the same places, and in the same way, with the fourth unit becoming a question. Alice Griffin's reading differed from that of T.J.B. Spencer's by offering the lighter comma in two instances where Spencer kept the semicolon, and by two additional commas in Spencer's, whereas mine differed from Griffin's by two commas, one in hers and 
one in mine in different places. The high level of coincidence suggests that readers must work through and around Riche's often misleading punctuation to find essentially the same groupings of words in order to make the clearest sense of what is, after all, a straightforward narrative relation. This is to suggest in turn that Elizabethan readers had to perform the same tacit editorial procedure in their minds in the production of common meanings. Yet I confess that there is some interpretation involved, and that interpretive component, no matter how slight, will set the purist kettle steaming.

Hence, the impasse will remain. Those committed to the notion of authenticity and the presentation of the book as a feature of meaning and interpretation will rationalize to the maximum the intentionality of all manner of early conventions and formatting. The editor concerned with the removal of every conceivable obstacle to a ready comprehension of the narrative logic and experience by the modern reader will seek to rationalize away the significance of all but the most compellingly substantive elements of the signing system, concentrating upon the story as a common experience of readers across time in accordance with the uniform and genetically determined features of mind whereby those narrative motifs are converted into reality-grounded meanings. The reason for the tempest is that texts cannot be presented both ways at once, while compromises and amalgamations of features are largely perplexing and meaningless. Texts are either mired in the circumstances of their earliest productions and must be encountered in those terms alone in acts of archaeological reconstruction, or they are relations of meaning to be dislodged from all the symbol systems in which they are presented, insofar as those systems are merely the means to 
cognitive and emotional representations. Where the latter approach is assumed, arguably the signing system should be made as unobtrusive and as familiar as possible. Neither position is without its problems, perhaps leaving the decision to personal taste. But in our world of blind vetting and prerequisite financing, the debate can take its toll in very material and professional ways. Because this divisive issue will always remain unresolved, and because editors must always choose one mode or another, subventions for editions of early modern editions become largely a matter of chance, for if a manuscript happens to meet with a proponent of the opposite view, its funding prospects are seriously diminished, largely because partiality on this score tends to colour every other aspect of the evaluation.

As a final thought, however, in an age of critical preferences prioritizing cultural negotiations and cognitive plasticity in conjunction with the history of the book wherein all inflections from the page become hermeneutic conditions, the harder case to be made is on behalf of the phylogenetic qualities of the mind that predetermine our common habits of meaning formation as a species. The "choses vues" of experience may seem random, personalized, constructed, and interpreted, but in matters of things in time and space, the external world has fine tuned the precision of our reception on the basis of evolutionary pressures within environments where we cannot afford, for long, to go wide off the mark. That is the beginning of interpretation. When information of that kind is presented through language, it is likewise governed by what we know of the world as a species through the engineered procedures of our cognitive orientation. Much of that experience is arranged for us by the processes of our own consciousness as narratives 
of minimal representations of personhood and events in an adequate setting - the foundational components of all storytelling. We have a noteworthy capacity to process such sequences as percepts (reality read through the senses) or as images (things created by the mind) on a nearly equal footing. Arguably, the constancy of these phenomena is not heavily determined by systems of writing and printing; phenomenological logic must shine through all such systems or the meaning of narrative dies. That there can be colourations of that meaning through variations in the sign systems conveying it may therefore be doubted. And to the extent that that is true, where primary narrative messages are concerned, the mediated text with the least obtrusive elements is the more authentic, so long as the true substantives of the original are honoured and glossed. Punctuation - the only arguably contestable feature - is a servant to narrative logic. If that logic remains the same from age to age, in that the Elizabethan mind is identical to our own as an instrument for negotiating environments, then the elements of narrative must have been fashioned in their minds in ways identical to our own, whatever the punctuation implies to the contrary. This argument in favour of the modernizing of texts as a courtesy to the reader, where the retention of earlier conventions and typefaces is indifferent to the generation of meaning, may not meet with ready acceptance, perhaps for want of extended analysis, but I think it is grounds for reflection. 


\title{
Bibliography
}

DAwson, Giles E. and Laetitia Kennedy-Skipton. 1966, Elizabethan Handwriting 1500-1650: A Manual, New York, W.W. Norton and Co.

DiCKEnSON, John. 2008, Greene in Conceit, eds Donald Beecher and David Margolies, Toronto, CRRS.

GRIFFIN, Alice, ed. 1966, The Sources of Ten Shakespearean Plays, New York, Thomas Crowell Co.

MCKerRow, R.B. 1967, An Introduction to Bibliography for Literary Students, Oxford, Clarendon Press.

Riche, Barnabe. 1992, A Farewell to Military Profession, ed. Donald Beecher, Binghamton, N.Y., MRTS.

Spencer, T.J.B. ed. 1968, Elizabethan Love Stories, Harmondsworth, Penguin Books.

\begin{abstract}
All Renaissance texts in modern editions must assume a format conforming to views concerning typefaces, spelling, and punctuation, whether of the initiating period or of the modern period. Representing the "other" is the watchword, and arguably this otherness is a component not only of Renaissance style and thought, but also of Renaissance printing conventions to the degree that they differ from our own. A rationale favouring the modernization of texts could however be in the very interests of authenticity.
\end{abstract}




\section{Résumé}

Les éditeurs des textes de la Renaissance présentés en éditions modernes doivent choisir entre les conventions typographiques des éditeurs d'origine ou une modernisation qui risque d'obscurcir certaines évidences précieuses pour favoriser une rencontre authentique avec le texte. Nous proposons ici le début d'une défense de l'édition modernisée. 